\title{
Using Cultural Historical Activity Theory (CHAT) to Frame 'SuperclubsPLUS', an Online Social Network for Children
}

\author{
Jennifer Masters \\ La Trobe University, Bendigo, Australia \\ j.masters@latrobe.edu.au
}

\begin{abstract}
This paper uses a Cultural Historical Activity Theory framework to describe a social-networking online community project, "SuperclubsPLUS", for children aged 6-12. The use of the CHAT frame enables a detailed description of connections within the project as participants work together to achieve individual and common goals. Application of this structure to the SuperclubsPLUS environment supports the concept that the community is continually changing, shaped by the interactions of the participants. It is anticipated that this snapshot of the project will provide a tangible base in order to further develop and map ongoing patterns of interaction for research.
\end{abstract}

Keywords: Cultural Historical Activity Theory, Online Social Networking, SuperclubsPLUS, cyber safety, cyber bullying.

\section{Introduction}

Cultural Historical Activity Theory (CHAT), also known simply as "Activity Theory", is a psychological theory that emerged in Russia in the 1920s based on the work of Lev Vygotsky. The core concept of this theory is that awareness emerges from an individual participating in a social structure where activity incorporating the use of tools to produce artifacts leads to socially valued outcomes. In doing so, the individual develops their own perspective, changing the way they think and behave in future situations. The CHAT model is represented by Engestrom [1] in the form of a triangle where the subject interacts with the community, rules, division of labour, the instruments and the object (artifact) to reach the outcome. This triangle has now become a common framework for representing understanding based on this theory (see Fig 1).

Cultural Historical Activity Theory has been used for over 20 years as a perspective for investigating a wide range of human activity and, because of its emphasis on artifacts, it is particularly suited to human-computer interactions (HCI) [2]. In an edited book, Nardi presents a number of applications of CHAT used for $\mathrm{HCI}$, ranging from the design of software interfaces to transformations of education communities through the use of ICT. Some other diverse examples where CHAT has been used to support HCI analysis include the use of computers in a remote Australian Indigenous school [3], an online community for mathematics and science teachers [4] and a Multimedia workshop for at risk children [5]. 


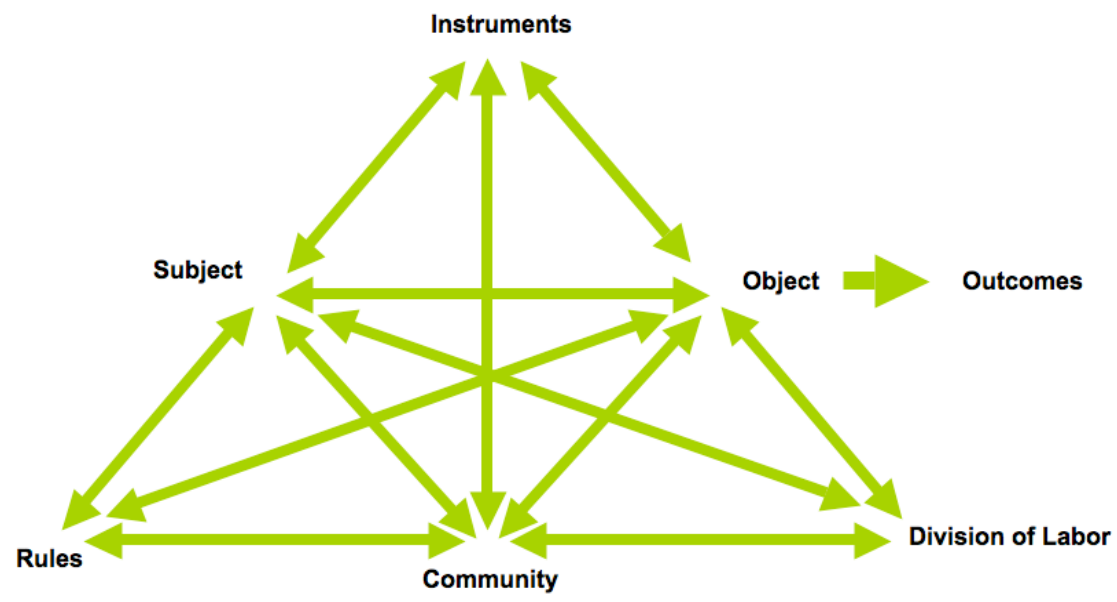

Fig. 1. The basic CHAT Triangle (based on Engstrom [1])

The SuperclubsPLUS project is intended to foster a rich environment where a number of research avenues will be investigated. A broad theoretical framework based on Activity Theory will be used as a global structure to represent the SuperclubsPLUS community and environment. As Cultural Historical Activity Theory not only provides a theoretical basis but also a guide to practice, the CHAT triangle is used for an analytical framework. In this context, the study considers the environment as an activity system and maps the interactions between participants (children, teachers and mediators), the technology, and the virtual environment. Understandings are drawn primarily from transcripts of online communication, but also virtual artifacts created by participants.

\section{What Is SuperclubsPLUS?}

SuperclubsPLUS is an online learning community for children 6-12 to participate in social networking. SuperclubsPLUS was an initiative of an organization called Intuitive Media that was founded by in 1998 in England. SuperclubsPLUS emerged in 2006 from a previous project known as GridClubs: SuperClubs and provides an online learning community for children to talk to current friends and meet new ones, publish and be creative, participate in forums and discussions and learn new ICT skills. SuperClubsPLUS is a safe environment because:

- All members are authenticated through their schools, and only children and teachers from registered schools can access the environment

- Teachers can see everything that their students write or create

- Highly trained mediators are rostered on to facilitate interactions and scaffold creative work and to actively protect the children

- Sophisticated content checking tools are used by the mediators to monitor all communications, protecting children from bullying or abuse. 
The program was very successful when it was introduced in the UK, and the English program has 120,000 children and 13,000 teachers as members. The Australian implementation is an initiative of Intuitive Media Australia in collaboration with La Trobe University, Victoria, with funding from the Telstra Foundation. SuperclubsPLUS was introduced in Australia in May 2008 and officially launched in September. At the end of March 2009 there were around 47,000 children participating in SuperclubsPLUS Australia, along with 3000 teachers. There are currently 18 trained Australian mediators who support the children online. It is anticipated that there will be 100,000 Australian participants within two years.

SuperClubsPLUS provides a rich environment for personalised and social learning. While the program may be implemented by a teacher as part of the school curriculum in a traditional sense, much of the interaction is informal with children (and teachers) participating in their leisure time in out of school contexts. This type of use is supported by the extended opening hours, including weekends. The children have full access to all facilities during 'Live Time', usually from 8.00am to 8.00pm and then 'Build Time' allows them to work on constructions such as articles, web pages or projects, but not communications at all other times.

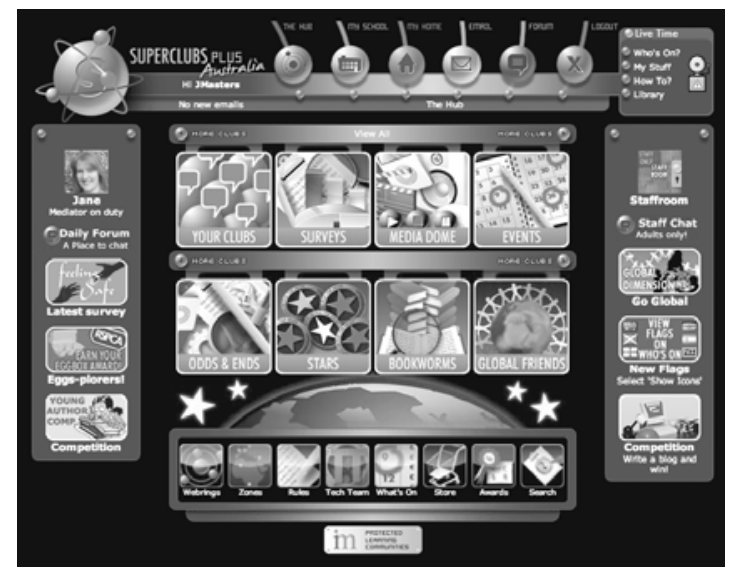

Fig. 2. The SuperclubsPLUS interface, showing the hub with the club activities and the mediator on duty

While the SuperclubPLUS environment is informal and free-form, there are plenty of activities to keep children engaged and involved. Children usually get started in SuperclubsPLUS by doing their "stars". There are five different coloured stars that are awarded to the children as they complete lists of ICT skill orientated tasks. When the tasks are completed satisfactorily, the star automatically appears on the child's personal web page. The first star, the white star, relates to safety online and requires the child to successfully complete a quiz before proceeding. The subsequent stars (red, green, blue and yellow) support the children to build their web pages within a template, construct email and contribute to forums, create and upload images and use 'BB' codes (a subset of html) to hone their page display. The children also participate in a wide range of forums. These can be play orientated, such as telling jokes or roleplay but can also be related to topic discussion, notably in the form of a "hotseat" where the children get to talk with an expert about their particular area of interest. 


\section{SuperclubsPLUS within a CHAT Frame}

As a contained virtual community, SuperclubsPLUS is an ideal candidate for framing within a Cultural Historical Activity Theory framework. Blanton et al. [6] identified that a CHAT context is determined by five principles. The first CHAT principle is that human behaviour is social in origin; the second principle is that human activity is mediated through tools; the third principle is the centrality of communication in activity; and the fourth principle the meaning of values, beliefs, and normative expectations, is brought about by the process of objectification. The last is the principle that learning and development are incorporated in the activities of communities of practice (p. 438-440). An important consequence of the activity within the system is that this activity brings about change to the very structure of the environment. This means that rather than being a static construction in which the activity takes place, the system is organic, grows and changes as objects are achieved.

In SuperclubsPLUS these principles are clearly evident. The genre is social networking and the online environment provides powerful tools to facilitate social connections, embedded in activities provided for members. Online communication in the form of publication, forums and email is the conduit for all interactions in SuperclubsPLUS and "objectification" is evident in the construction of artefacts that members strive for, either displayed in personal web pages or published in communal spaces - the clubs. Learning and development are underlying concepts that drive SuperclubsPLUS. While learning is quite often informal and is initiated by the learner rather than an external influence, eg. a teacher, the collective growth of knowledge and content in SuperclubsPLUS truly establishes its status as a dynamic and vibrant activity system. In order to use the CHAT structure for analysis in this context, the SCP environment needs to be represented in the context of the CHAT triangle.

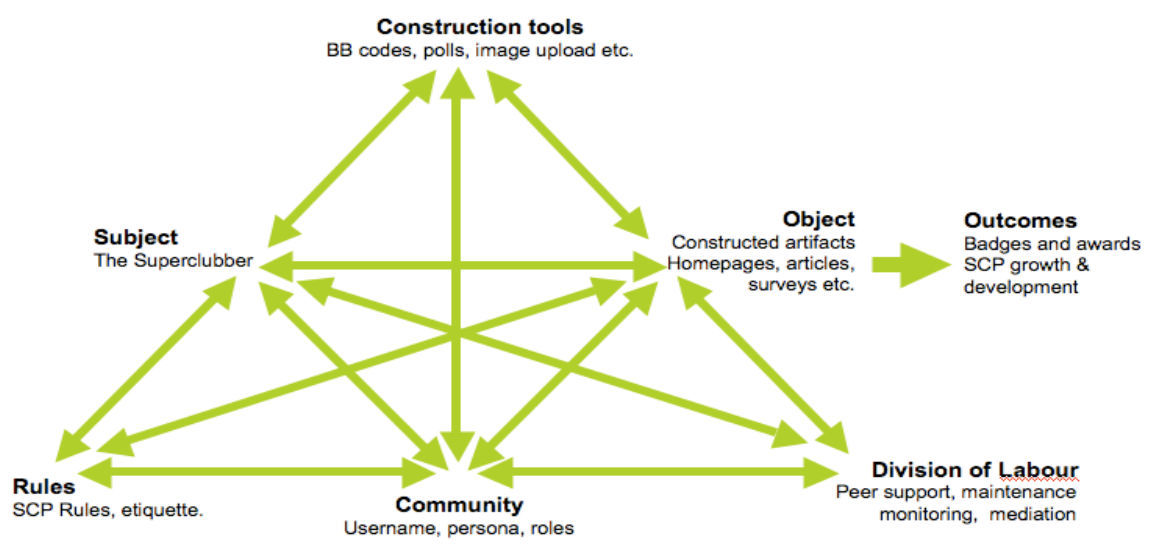

Fig. 3. The SuperclubsPLUS environment in a CHAT context

\subsection{Subject}

The subject is the participant in the community. In most contexts this refers to an individual but may also refer to a group [7]. In SuperclubsPLUS everyone who is 
subscribed to the community is a subject - a "Superclubber". While some of these participants may have roles beyond this base status, every subscriber has, by default, a password and a "My Home" space where they can build a profile. This means that every participant, including the teacher, can play the role of the subject.

\subsection{Construction Tools}

In some representations of the CHAT triangle, "instruments" are listed as "tools", "means", and "artifacts" or even mediating tools/means/artefacts [6]. In the SCP environment, "tools" probably serves as a better metaphor. The tools used for SCP could be considered on a technical level and include the computer or perhaps a mobile device such as an iPhone and the software, the Internet browser and the ICE engine1 that is used to power SuperclubsPLUS. For this analysis though, it is perhaps more valuable to consider the tools within the SuperclubsPLUS environment used to scaffold the Superclubbers' construction processes, nominally "construction tools". For this purpose we can include devices such as the edit mechanisms on the home pages, the BB codes that the students to publish text, quizzes, emails and checklists (that are used to trigger award processes), article and library upload mechanisms and forums.

\subsection{Objects}

"Object" refers to the object of the activity. It can means goals or purpose, often leading to the construction of an artefact. Roth [7] identifies that the object may represent a desired state where the subject works towards achievement. When the goal state is reached the object represents the outcome and the subject is able to set new goals bringing about change to their position and consequently changing the dynamics of the community, even if it is at a microscopic level. The SuperclubsPLUS environment provides a context where objects are tangible and accessible for community members on a number of layers. Although Superclubbers are not prescribed activity, the environment provides a wide range of activities, tasks and challenges where participants can work towards goals they select. Some of the objects in SuperclubsPLUS include the development of home pages that represent the owner in aspects such as hobbies, interests, musical and sporting preferences, achieving the Star awards and additional awards, creation of project pages and publishing of articles. Superclubbers can also set more immediate goals for themselves. These include outcomes such as making new friends via email, getting other Superclubbers to visit their page, and participating in one of the many forum activities where they can contribute to discussions on current topics and play in scenarios, such as the virtual pet shop.

\subsection{Rules}

The SuperclubsPLUS community is governed by a set of ten clearly defined rules. These rules provide a charter for all members of the SuperclubPLUS community and include codes of conduct to prevent intimidation or cyber bullying, aspects of cyber safety relating to sharing personal details (including passwords and contact information), engaging in unsafe practices on any website and respecting copyright. 
The rules are used as the basis for mediation of interactions. Any communication is constantly monitored and the mediators will intercept any discussion or a publication that infringes the defined rules. Further, community members are asked to be actively involved in practising these rules and are encouraged to contact a mediator if they feel that the rules have been compromised.

In addition to the formal SCP rules, Superclubbers will encounter a number of processes and policies that they will need to abide by. These include aspects such as technical requirements, eg. the format and size of images, etiquette, such as only making a single entry per post in repetitive games (eg "count to a million") and publishing requirements for particular forums. In SuperclubsPLUS the rules and regulations of the environment help to shape a functional and dynamic society in which all members feel safe, valued and able to explore and build their understandings and confidence.

\subsection{Community}

In SuperclubsPLUS each community member has an icon that represents their online presence. Every member selects his or her own icon and this image can be changed to any icon in the library whenever desired. There isn't a problem with Superclubbers having the same icon and this happens quite often. In one forum it was noticed that two different Superclubbers had an identical icon - a flashing square with a logo reading, "I am unique"! The icon is always displayed next to the SCP username. Children are allocated their first name, the initial of their surname and a number that increments for the number of users with that particular combination. For example, AmyP82 is the 82nd user to have the first name "Amy" and the initial "P". Teachers are allocated their title and surname for a username "Mr Walsh", and they also have a small $\mathrm{T}$ symbol next to their name. A mediator is given their first name and their surname and has an $\mathrm{M}$ symbol.

While it is easy to distinguish between children, teachers and mediators, at the basic level all are Superclubbers and have a similar persona online. A Superclubber is represented by a homepage where he or she can post images and information and host polls and guest books. Each page has a user statistics bar across the top. On the left side, it displays the page title, username and icon and the affiliated school. It also has a gender icon; pink for girls, blue for boys, that can be activated if the user desires (see Fig 4).

When Superclubbers start out they have a single page to work with, however, as they earn stars and complete projects they can increase the number of page they can have to use. The status of a child Superclubber remains much the same while they increase stars, awards, page numbers and visitors to their page until they become eligible for the Tech Team. Children can apply to join the Tech Team when they have all their stars, have a well designed home page, have had over 200 visitors to their page, know the rules thoroughly and haven't received any warnings from the mediators for breaking the rules in the previous 6 months. When they achieve Tech Team status, a TT icon appears next to their username. 


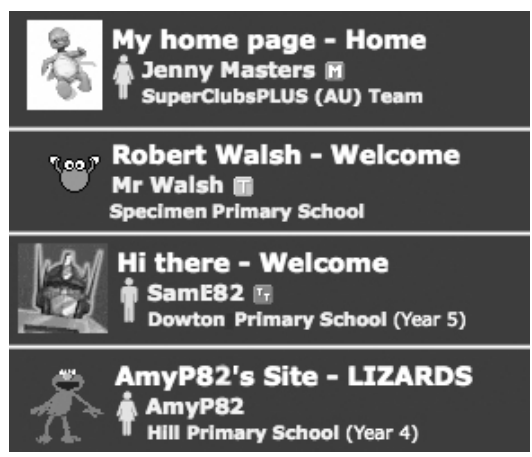

Fig. 4. Examples of the SuperclubsPLUS Profiles displayed on home pages

A teacher in SuperclubsPLUS has similar interactions with the SuperclubsPLUS tools and activities, with a notable difference in terms of communications. Teachers can email all students in their school but are not able to email children in any of the other SuperclubsPLUS schools. There are forums for teachers to talk to each other but teachers can't contribute to most of the children's clubs or forums. The only exception is the "Hotseats" forum where both children and teachers can post to the forum to ask the guest questions. A mediator is able to access all aspects of SuperclubsPLUS. They also have access to the mediator's area powered by the ICE Engine where email monitoring, image uploads and other mediating tasks are performed.

\subsection{Divisions of Labour}

At a cursory glance it may be possible to mistake SuperclubsPLUS as a "virtual school" environment where children interact with each other and adults - teachers and others - a space where adults tell children what to do. This, however, is a superficial perspective and does not reflect the true interactions in the space. In SuperclubsPLUS the children really own the environment. While mediators and teachers can suggest activity, the children decide on content, avenues of play and the communication they will have with others. The "work" that emerges through SuperclubsPLUS activity is primarily individual but often collaborative as children support each other to gain stars, critique each others web pages and participate in collaborative activities in forums. The division of labor is complicated, yet subtle and is allocated through negotiation, often scaffolded by the mediators. In this environment the teacher's role can be quite different.

The teacher may do fairly traditional roles such as maintain the school web site, which includes profiling the school and selecting children's work for publication. They may also check children's project pages and award badges for particular achievements. However, it is possible for the teacher to simply be a Superclubber, albeit a restricted member because they can't email to everyone who is online. In this situation the teacher is able to explore building pages, do their stars, complete surveys and complete special activities, just as the children do. 
The mediator's role is more like a party host than a police officer. When a mediator is on duty, he or she is there for the Superclubbers. The duty mediator's name and photograph is displayed on the front page and they can be emailed with a simple click. Superclubbers can ask questions, report problems or simply have a chat at any time. The Duty Mediator also monitors the emergency bell. This is displayed on every SuperclubPLUS page and has priority over every other duty. The other roles of the duty mediator include monitoring the forums, approving image submissions, and checking emails. At busy times more than one mediator will be rostered on, and the duties will be shared, with the lead or "Heads Up" mediator orchestrating this process. Mediators are often online when they are not on duty. In these instances the mediator may be doing other roles like approving articles for publication or they can be working on their own skills and resources, basically being a Superclubber. At these times the mediator can choose to be "hidden" or "visible" to the children.

\subsection{Outcomes}

The outcomes in SuperclubsPLUS can be examined at an individual level but also at a community level. In terms of an individual, outcomes are represented iconically through the use of badges and awards (see Fig 5).

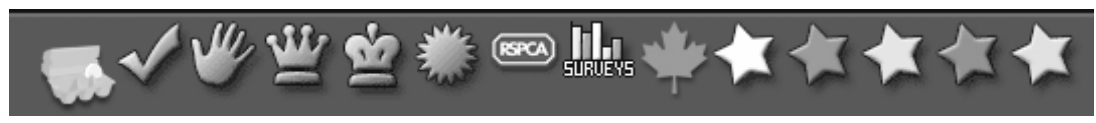

Fig. 5. Badges and awards on a SuperclubsPLUS homepage

These icons are truly representative of objects achieved because in many instances a Superclubber will remove a product once the award is achieved in order to reuse the page allocated on the home site. For example, a Superclubber must, amongst other things, upload a particular image of "egg on toast" to earn their blue star. Once the star has been awarded the image can be removed from the page. The object is no longer present but the outcome is evident through the blue star icon.

While the outcomes at a community level are less tangible, they are much more profound than the visual symbols as they represent the growth of the SuperclubsPLUS as a dynamic and vibrant community. Community outcomes in SuperclubsPLUS are represented by the transforming environment, created by the constant tweaking of functions, new activities and processes and better and more efficient ways to achieve the communities' desire for objects.

\section{Summary}

While Cultural Historical Activity Theory can be frustratingly multi-dimensional, the premise that activity cannot be understood without understanding the way artifacts are integrated through social practice [2] has been a useful principle for the studying the SuperclubsPLUS environment. The components of the CHAT triangle have provided an effective lens for examining interactions in the community and the mapping process highlights the complexities and the nuances in the roles, tasks and 
relationships in the community. For example, this frame has been used for examining the project as a mechanism to teach children about "cybercitizenship", where the potential of the adult mediation to develop online social behaviour, especially in the context of preventing cyber bullying, is discussed [8].

The mapping activity described in this report captures a moment in time for SuperclubsPLUS. Through the process of completing this task, the nature of the community as an organic entity becomes obvious. The community is constantly changing as Superclubbers explore new options, meet new people and achieve new goals. The SuperclubsPLUS production team is consistently looking for options to provide more material - construction tools and tasks for the participants and mediation mechanisms for the mediators. It is anticipated that the adults in SuperclubsPLUS, the mediators but also the teachers, will progressively become more experienced at working in SCP and therefore capitalize more on the educational opportunities provided. Conversely children in SuperclubsPLUS will become more skilled and sophisticated in their activity and it is likely the balance will change where more children take on the TechTeam role to support novice Superclubbers.

Regular mapping of the SuperclubsPLUS community within the CHAT framework will help to establish a familiar understanding for those who are working in the environment. This exercise will also contribute to our understandings of social networking, particularly in regards to children developing an awareness of practices and principles over a period of time.

\section{References}

1. Engeström, Y.: Learning by Expanding: An Activity-theoretical Approach to Developmental Research. Orienta-Konsultit, Helsinki (1987)

2. Nardi, A. (ed.): Context and Consciousness: Activity Theory and Human-computer Interaction. MIT Press, Cambridge (1996)

3. Lloyd, M., Cronin, R.: A Community of Teachers: Using Activity Theory to Investigate the Implementation of ICTE in a Remote Indigenous School. Presented at AARE 2002, Brisbane, Queensland, December 1-5 (2002)

4. Barab, S., Schatz, S.: Scheckler: Using Activity Theory to Conceptualize Online Community and Using Online Community to Conceptualize Activity Theory. Mind, Culture, and Activity 11(1), 25-47 (2004)

5. Betts, J.D.: Multimedia Arts Learning in an Activity System: New Literacies for At Risk Children. International Journal of Education \& the Arts 7(7) (2006)

6. Blanton, W., Simmons, E., Warner, M.: The Fifth Dimension: Application of CulturalHistorical Activity Theory, Inquiry-Based Learning, Computers, and Telecommunications to Change Prospective Teachers' Preconceptions. Journal of Educational Computing Research 24(4), 435-463 (2001)

7. Roth, W.: Activity Theory and Education: An Introduction. Mind, Culture, and Activity 11(1), 1-8 (2004)

8. Masters, J., Yelland, N.: Changing Learning Ecologies: Social Media for Cyber-citizens. In: Shariff, S., Churchill, A. (eds.) Truths and Myths of Cyber-bullying: Stakeholder Responsibility and Children's Safety, Peter Lang, New York (2009) 